\title{
PSEUDO-ARCS AND CONNECTEDNESS IN HOMEOMORPHISM GROUPS
}

\author{
WAYNE LEWIS
}

\begin{abstract}
We prove that the homeomorphism group of the pseudo-are contains no nondegenerate continua. An equivalent result holds for any continuum all of whose small subcontinua are pscudo-arcs. e.g. for the pseudo-circle and the circle of pseudo-ares. Related questions are briefly mentioned
\end{abstract}

A continuum is a compact, connected metric space. The pseudo-arc is a chainable, hereditarily indecomposable continuum [M]. It is also homogeneous [B]. The space of homeomorphisms of a continuum. topologized by the compact open topology. is a complete separable metric topological group. (Though the sup metric induces this topology, it is in general not complete.)

For manifolds it is known that this group always contains Hilbert space factors. In general, however, very little is known about the topological structure of homeomorphism groups (e.g. connected subsets, dimension), even for well-studied spaces. The pseudo-arc is of course very far from being a manifold. but in some ways has very nice structure about which quite a bit is known. and it has been the subject of considerable interest.

Beverly Brechner has asked $[\mathbf{B r} \mathbf{l}]$ whether the homeomorphism group of the pseudo-arc contains any nondegenerate continua - in particular, whether it contains copies of the pseudo-arc. We provide a negative answer to this question.

We will use the following theorem. the proof of which is left to the reader.

Throrem. Let $X$ and $Y$ be continua, $Y$ chainable. Let $f, g: X \rightarrow Y$ he maps such that $f(X) \supset g(X)$. Then there exists $x \in X$ such that $f(x)=g(x)$.

We are now ready to prove the claimed result.

THEOREM. The space of homeomorphisms of the pseudo-arc contains no nondegenerate continua.

Proof. Let $P$ be the pseudo-arc. Suppose $M$ is a nondegenerate continuum in the space of homeomorphisms of $P$. Then $M$ induces a homeomorphism $h: P \times M \rightarrow P$ $\times M$ defined by $h(x, y)=(y(x), y)$. Let $\pi: P \times M \rightarrow P$ be projection onto the first coordinate. We may assume without loss of generality that $M$ contains the identity.

Received by the editors April 26, 1982 and. in revised form. August 4. 1982. Thene results will be presented at the A. M. S. meeting in Baton Rouge. Louisiana. November 12-13. 1982

1980 Mathemathes Suhject (lassificatım. Primary 54F20): Secondary 54H15.

Key words and phrases. Continuum, pseudo-are, homeomorphism group. homogencits. hereditar! indecomposability. 
There exists $y \in M, y \neq$ identity, and $x_{0} \in P$ such that $y\left(x_{0}\right) \neq x_{0}$. Let $y_{0}=y\left(x_{0}\right)$. Then $y_{0} \in \pi h\left(\left\{y_{0}\right\} \times M\right) \cap \pi h\left(\left\{x_{0}\right\} \times M\right) \neq \varnothing$. Since $P$ is hereditarily indecomposable and $\pi h\left(\left\{y_{0}\right\} \times M\right)$ and $\pi h\left(\left\{x_{0}\right\} \times M\right)$ are two intersecting subcontinua, either $\pi h\left(\left\{y_{0}\right\} \times M\right) \subset \pi h\left(\left\{x_{0}\right\} \times M\right)$ or $\pi h\left(\left\{x_{0}\right\} \times M\right) \subset \pi h\left(\left\{y_{0}\right\} \times M\right)$. Suppose $\pi h\left(\left\{y_{0}\right\} \times M\right) \subset \pi h\left(\left\{x_{0}\right\} \times M\right)$.

Define $f_{x_{0}}: M \rightarrow P \times M$ by $f_{x_{0}}(z)=\left(x_{0}, z\right)$ and $f_{y_{0}}: M \rightarrow P \times M$ by $f_{v_{1}}(z)=$ $\left(y_{0} . z\right)$. Then $\pi h f_{x_{0}}$ and $\pi h f_{y_{0}}$ are two maps from $M$ into $P$ with $\pi h f_{v_{0}}(M) \subset \pi h f_{x_{10}}(M)$. Thus there exists $z_{0} \in M$ such that $\pi h f_{v_{0}}\left(z_{0}\right)=\pi h f_{x_{0}}\left(z_{0}\right)=p_{0}$.

This means that $h f_{y_{0}}\left(z_{0}\right)=h\left(y_{0}, z_{0}\right)=\left(p_{0}, z_{0}\right)=h\left(x_{0}, z_{0}\right)=h f_{x_{0}}\left(z_{0}\right)$. But $h$ is a homeomorphism and $\left(y_{0}, z_{0}\right) \neq\left(x_{0}, z_{0}\right)$. This is a contradiction, and thus $M$ must be degenerate.

Suppose $X$ is a continuum all of whose nondegenerate small subcontinua are pseudo-arcs. Then an argument similar to the above can be used. restricting to subcontinua of $M$ small enough so that the tracks of points stay inside these pseudo-arcs. to show that the space of homeomorphisms of $X$ contains no nondegenerate continua. Such spaces include the circle of pseudo-arcs and the pseudo-circle. Thus we have the following corollary.

COROllary. If $X$ is a continuum with the property that for some $\varepsilon>0$ all subcontinua $K$ of $X$ with $0<\operatorname{diam}(K)<\varepsilon$ are pseudo-arcs, then the space of homeomorphisms of $X$ contains no nondegenerate continua.

We have shown that every compact subset of the homeomorphism group of the pseudo-arc is zero dimensional. We do not know whether this homeomorphism group contains any nondegenerate noncompact connected subsets.

Question 1 ( B. Brechner). Is the homeomorphism group of the pseudo-arc totally disconnected?

Question 2. Is the homeomorphism group of every hereditarily indecomposable continuum totally disconnected?

There is no homogeneous continuum whose homeomorphism group is known to contain a nondegenerate connected set where the homeomorphism group is not also known to contain an arc.

Question 3. Must the homeomorphism group of a homogeneous continuum either contain an arc or be totally disconnected?

Our result gives no information about the dimension of the homeomorphism group of the pseudo-arc. For the nondegenerate homogeneous continua for which the dimension of their homeomorphism group is known. that group is infinite dimensional.

Question 4. Is the homeomorphism group of the pseudo-arc infinite dimensional?

Question 5. Is the homeomorphism group of every nondegenerate homogeneous continuum infinite dimensional?

The Menger universal curve has a totally disconnected homeomorphism group [Br2], and one can show further that no nondegenerate connected subset of the space of continuous maps of the Menger curve into itself contains a homeomorphism [L]. (This space of maps does, however, contain nondegenerate connected subsets.) 
Question 6. Is every connected subset of the space of continuous maps of the pseudo-arc into itself which contains a homeomorphism degenerate?

Using this above property about the space of maps of the Menger universal curve $M$ into itself, and examining what happens to fibers $\{m\} \times X$ under certain homeomorphisms of $M \times X(X$ a nondegenerate continuum), we showed [L] that $M \times X$ is Galois if and only if $X$ is isotopy Galois. (A space $X$ is Galois [FS] if for each $x \in X$ and open $U$ containing $x$ there is a homeomorphism $h: X \rightarrow X$ with $h(x) \neq x$ and $\left.h\right|_{X-U}=\mathrm{id}_{X-U} . X$ is isotopy Galois [DFM] if for each $x \in X$ and open $U$ containing $x$ there is an isotopy $F: X \times[0,1] \rightarrow X$ with $F((y, 0))=y$ for each $y \in X, F((x, 1)) \neq x$, and $F((y, t))=y$ for each $y \notin U$ and $t \in[0,1]$.)

The similarity of that argument to the result presented here suggests, but by itself does not seem enough to answer, the following question.

Question 7. If $P$ is the pseudo-arc and $X$ is a nondegenerate continuum. is $P \times X$ Galois if and only if $X$ is isotopy Galois?

Our argument shows that some restrictions are imposed on the motions of fibers under certain homeomorphisms of the square of the pseudo-arc. From similar considerations, it is known [KKT] that if $h$ is a homeomorphism of $\prod_{\alpha \in A} M_{\alpha}$, where each $M_{\alpha}$ is either the Menger universal curve or the Sierpinski universal curve, then $h=\prod_{\alpha \in A} h_{s(\alpha)}$, where $s$ is a permutation of $A$ and $h_{s(\alpha)}$ is a homeomorphism of $M_{\alpha}$ onto $M_{s(\alpha)}$.

Question 8. If $h$ is a homeomorphism of $\prod_{\alpha \in A} P_{\alpha}$, where each $P_{\alpha}$ is a pseudo-arc, is $h$ necessarily of the form $h=\prod_{\alpha \in A} h_{s(\alpha)}$, where $s$ is some permutation of $A$ and $h_{s(\alpha)}$ is a homeomorphism of $P_{\alpha}$ onto $P_{s(\alpha)}$ ?

W. Kuperberg has defined a space $X$ to be pseudo-contractible if there exists a continuum $C$, two points $a$ and $b$ of $C$, and a map $F: X \times C \rightarrow X$ such that $F((x, a))=x$ and $F((x, b))=y$ for some fixed $y \in X$ and each $x \in X$. He then asks the following (Problem 31, University of Houston Problem Book)

Question 9 ( $W$. Kuperberg). Is it true that the pseudo-arc is not pseudo-contractible?

This is equivalent to answering Question 6 above in the case of a continuum $C$ connecting the identity to a constant map. While our result does not answer this the techniques here can be used to show that if $K$ is a subcontinuum of $C$ and $P^{\prime}$ a subcontinuum of the pseudo-arc $P$ then either all the levels of the pseudo-contraction corresponding to points of $K$ agree on $P^{\prime}$ or else one of these levels fails to be one-to-one when restricted to $P^{\prime}$.

\section{REFERENCES}

[B] R. H. Bing, A homogeneous indecomposable plane continuum. Duke Math. J. 15 (1948), 729-742

[Br1] Beverly Brechner, Homeomorphism groups of chainable and homogeneous continua. Proc. Texas Topology Summer 1980, Austin (to appear).

[Br2] . On the dimensions of certain spaces of homeomorphisms. Trans. Amer. Math. Soc. 121 (1966), 516-548.

[DFM] Paul F. Duvall, Peter Fletcher and Robert A McCoy, Isotopy Galois spaces, Pacific J. Math. 45 (1973), 435-442.

[FS] P. Fletcher and R. L. Snider, Topological Galois spaces, Fund. Math. 68 (1970), 143-148. 
[KKT] K. Kuperberg. W. Kuperberg and W. R. R. Transue. On the 2-homogeneity of ('artesian products. Fund. Math. $110(1980), 131-134$.

(L) Wayne Lewis. Homeomorphism groups and homogeneous comtinua. Topology Proc. 6 (1981). $335-344$.

[M] E. E. Moise, An indecomposahle plane continuum which is homeomorphic to cach of its nondegenerate subcomtinua. Trans. Amer. Math. Soc. 63 (1948). 581-594.

Department of Mathematics. University OF KentuCKy. Li:Xington. Kintucky 40506

Current address: Department of Mathematics. Texas Tech University, Lubbock. Texas 794()9 